\title{
Direct Evaluation of the Quark Content of Nucleons from Lattice QCD at the Physical Point
}

\author{
A. Abdel-Rehim, ${ }^{1}$ C. Alexandrou, ${ }^{1,2}$ M. Constantinou, ${ }^{1}$ K. Hadjiyiannakou, ${ }^{1,2}$ K. Jansen, ${ }^{3}$ \\ Ch. Kallidonis, ${ }^{1}$ G. Koutsou, ${ }^{1}$ and A. Vaquero Avilés-Casco ${ }^{4}$ \\ ${ }^{1}$ Computation-based Science and Technology Research Center, The Cyprus Institute, 20 Kavafi Street, Nicosia 2121, Cyprus \\ ${ }^{2}$ Department of Physics, University of Cyprus, P.O. Box 20537, 1678 Nicosia, Cyprus \\ ${ }^{3}$ NIC, DESY, Platanenallee 6, D-15738 Zeuthen, Germany \\ ${ }^{4}$ INFN Sezione di Milano-Bicocca, Edificio U2, Piazza della Scienza 3, 20126 Milano, Italy \\ (Received 9 January 2016; revised manuscript received 18 May 2016; published 23 June 2016)
}

\begin{abstract}
We evaluate the light, strange, and charm scalar content of the nucleon using one lattice QCD ensemble generated with two degenerate light quarks with mass fixed to their physical value. We use improved techniques to evaluate the disconnected quark loops to sufficient accuracy to determine the strange and charm nucleon $\sigma$ terms in addition to the light quark content $\sigma_{\pi N}$. We find $\sigma_{\pi N}=37.2(2.6)\left(\begin{array}{c}4.7 \\ 2.9\end{array}\right) \mathrm{MeV}, \sigma_{s}=41.1(8.2)\left(\begin{array}{l}7.8 \\ 5.8\end{array}\right) \mathrm{MeV}$, and $\sigma_{c}=79(21)\left(\begin{array}{c}12 \\ 8\end{array}\right) \mathrm{MeV}$, where the first error is statistical and the second is the systematic error due to the determination of the lattice spacing, the assessment of finite volume, and residual excited state effects.
\end{abstract}

DOI: 10.1103/PhysRevLett.116.252001

Introduction.-The scalar quark contents of the nucleon, or the so-called nucleon $\sigma$ terms, are fundamental quantities of QCD determining the mass generated by the quarks in the nucleon and thus related to the explicit breaking of chiral symmetry. They are relevant for a wide range of physical processes, such as pion- and kaon-nucleon scattering, but also for the interpretation of direct-detection dark matter searches being undertaken by a number of experiments [1]. Dark matter candidates under consideration are weakly interacting massive particles (WIMPs) predicted in many beyond the standard model theories that interact with normal matter by elastic scattering with nuclei [2]. In such a process, a WIMP, due to its large mass, produces a Higgs boson that interacts with the nucleon via scalar density operators. At zero momentum transfer, the cross section for spin independent elastic scattering depends quadratically on the nucleon scalar matrix element, which constitutes the largest uncertainty [3].

It is customary to define the nucleon $\sigma$ terms to be scheme- and scale-independent quantities:

$\sigma_{f}=m_{q_{f}}\left\langle N\left|\bar{q}_{f} q_{f}\right| N\right\rangle, \quad \sigma_{\pi N}=m_{u d}\langle N|\bar{u} u+\bar{d} d| N\rangle$,

for a given quark $q_{f}$ of flavor $f$, or for the isoscalar combination, where $m_{q_{f}}$ is the mass of $q_{f}, m_{u d}=\left(m_{u}+m_{d}\right) / 2$ is the average light quark mass, and $|N\rangle$ is the nucleon state. Since the pioneering chiral perturbation theory analysis that yielded $\sigma_{\pi N} \sim 45 \mathrm{MeV}$ [4], there has been significant progress in the determination of $\sigma_{\pi N}$ from experimental data $[5,6]$. Using high-precision data from pionic atoms to determine the $\pi N$-scattering lengths and a system of RoySteiner equations that encode constraints from analyticity, unitarity, and crossing symmetry a value of $59.1(3.5) \mathrm{MeV}$ is obtained [7]. This larger value of $\sigma_{\pi N}$ has theoretical implications on our understanding of the strong interactions as stressed in Ref. [8]. The determination of the strange $\sigma_{s}$ term is more difficult since it would require an analysis of kaonnucleon scattering phase shifts. Alternatively, one can use as input the values of $\sigma_{\pi N}$ and of the ratio $m_{s} / m_{u d}$, and SU(3) chiral perturbation theory to determine it. The evaluation of the charm scalar content is even harder amplifying even further the uncertainty in the WIMP-nucleon cross section.

Given the importance of these quantities, a number of lattice QCD calculations have been undertaken using two approaches [9]. The first uses the Feynman-Hellmann theorem that is based on the variation of the nucleon mass $m_{N}$ with $m_{q_{f}}: \sigma_{f}=m_{q_{f}}\left(\partial m_{N} / \partial m_{q_{f}}\right)$. Within lattice QCD the mass of the nucleon has been computed using various discretized actions and different values of the light quark mass including recent simulations with the physical value (referred to as the physical point). The most extensive analysis, using a large set of simulations with two light and a strange clover-improved fermions reaching physical values of the pion mass and including an assessment of finite volume effects and an extrapolating to the continuum limit, yielded a precise value of $\sigma_{\pi N}=38(3)(3) \mathrm{MeV}$ [10]. However, using the same approach to evaluate $\sigma_{s}$ yields larger errors. The analysis of Ref. [10] finds $\sigma_{s}=105(41)(37) \mathrm{MeV}$, while for $\sigma_{c}$ this approach is currently not applicable.

An alternative method is to evaluate directly the nucleon matrix elements of the scalar operator that involves disconnected quark loops. These are much more demanding to compute than hadron masses. Therefore, it is only recently that a direct computation of the $\sigma$ terms has been performed using dynamical simulations [11-16]. In this Letter we employ improved methods to compute the disconnected 
quark loops, and evaluate $\sigma_{\pi N}, \sigma_{s}$, and $\sigma_{c}$ directly at the physical point, thus eliminating chiral fits. The evaluation is done for one ensemble of $N_{f}=2$ twisted mass fermions at maximal twist with a lattice spacing of $a=0.093(1) \mathrm{fm}$ determined from the nucleon mass [17]. An analysis using a single ensemble precludes the evaluation of cutoff and infinite-volume effects using directly lattice QCD results on these quantities. However, we present conservative estimates of these systematics using auxiliary arguments.

Since we are using an $N_{f}=2$ ensemble, the strange and charm quarks are absent from the sea. Results obtained using $N_{f}=2$ and $N_{f}=2+1+1$ twisted mass ensembles on quantities for which such quenching can potentially have a large effect, such as the strange and charm quark masses $[18,19]$ or the kaon and $D$-meson decay constants $[20,21]$, showed no detectable quenching effects. For the value of $\sigma_{s}$ itself, there are two direct computations of the matrix element using the same overlap fermion ensembles, one with $N_{f}=2$ [22] and one with $N_{f}=2+1$ [23] both yielding consistent values albeit with a large systematic uncertainty. A more recent direct evaluation of the matrix element with overlap valence quarks on $N_{f}=2+1$ domain wall sea fermions including the physical point yielded a value $\sigma_{s}=32.3(4.7)(4.9)$, which is compatible with the value of $\sigma_{s}=30(8)(21) \mathrm{MeV}$ obtained with $N_{f}=2$ overlap fermions [22]. These results suggest that quenching effects are negligible within the statistical accuracy that they are presently computed. We, therefore, neglect quenching effects in this Letter.

Matrix elements in lattice $Q C D$.- The nucleon scalar matrix element can be extracted from the ratio of the threepoint function of the scalar operator to the two-point function at zero momentum transfer, $R\left(t_{s} ; t_{\text {ins }}\right)=\left[C_{3 \mathrm{pt}}\left(t_{s}-\right.\right.$ $\left.\left.t_{0}, t_{\text {ins }}-t_{0}\right) / C_{2 \mathrm{pt}}\left(t_{s}-t_{0}\right)\right]$ [17]. Inserting a complete set of states yields

$$
\begin{aligned}
& R\left(t_{s}, t_{\text {ins }}\right)=\frac{\sum_{i, j=0}^{\infty} A_{i j} e^{-\delta E_{i}\left(t_{s}-t_{\text {ins }}\right)} e^{-\delta E_{j}\left(t_{\text {ins }}-t_{0}\right)}}{1+\sum_{i=1}^{\infty} c_{i} e^{-\delta E_{i}\left(t_{s}-t_{0}\right)}} \\
& \underset{\delta E_{i}\left(t_{s}-t_{\text {ins }}\right) \gg 1}{\delta E_{i}\left(t_{\text {ins }}-t_{0}\right) \gg 1} m_{q_{f}}\left\langle N\left|\bar{q}_{f} q_{f}\right| N\right\rangle,
\end{aligned}
$$

where $\delta E_{i}=E_{i}-E_{0}$ is the energy gap between the $i$ th nucleon excited state $E_{i}$ and the ground state $E_{0}=m_{N}$, $A_{00}$ is the desired matrix element, $O=m_{q_{f}} \bar{q}_{f} q_{f}$, and we consider the light, the strange, and the charm quark flavors. In Eq. (2) the desired matrix element is obtained when the insertion-source, $t_{\text {ins }}-t_{0}$, and the sink-insertion, $t_{s}-t_{\text {ins }}$ time separations are large enough so that contributions to the matrix element of $O$ from excited states with the same quantum numbers as the nucleon are negligible. For the scalar operator these have been shown to be large [14]. We use three approaches in order to check that indeed excited state contributions are sufficiently suppressed. (i) In the socalled plateau method we look for the range of the values of $t_{\text {ins }}$ for which the ratio of Eq. (2) becomes time independent (plateau region) and then fit a constant within this region. This is done for several values of $t_{s}$. Excited states are sufficiently suppressed when the value of the plateau remains statistically unchanged. Smearing techniques are crucial to reduce the coefficients $A_{i j}$ for $i, j>0$ and $c_{i}$ in the three- and two-point functions. We use both Gaussian and APE smearing to maximize the overlap of our interpolating field with the nucleon [17]. (ii) In the summation method [24] we consider the ratio of Eq. (2) summed over $t_{\text {ins }}$ :

$$
R^{\mathrm{sum}}\left(t_{s}\right)=\sum_{t_{\mathrm{ins}} / a=1}^{t_{s} / a-1} R\left(t_{s}, t_{\mathrm{ins}}\right)=\frac{\sum_{i=0}^{\infty} A_{i i}\left(t_{s} / a-2\right) e^{-\delta E_{i} t_{s}}+\sum_{i \neq j=0}^{\infty} A_{i j} \frac{e^{-a \delta E_{i j}}-e^{-\delta E_{i j} t_{s}}}{1-e^{-a \delta E_{i j}}} e^{-\delta E_{i} t_{s}}}{1+\sum_{i=1}^{\infty} c_{i} e^{-\delta E_{i} t_{s}}}
$$

where we set $t_{0}=0$ for simplicity in what follows and define $\delta E_{i j}=E_{j}-E_{i}$. For large enough $t_{s}, R^{\text {sum }}$ depends linearly on $t_{s} / a$, and the matrix element is given by $A_{00}$, obtained as the slope of a linear fit. While excited state contributions are suppressed as $e^{-\delta E_{1} t_{s}}$, as compared to $e^{-\delta E_{1}\left(t_{s}-t_{\text {ins }}\right)}$ and $e^{-\delta E_{1} t_{\text {ins }}}$ in the plateau method, fitting to a two-parameter linear dependence increases the statistical errors. (iii) In the so-called two-state fit, one takes into account the contributions of the first excited state by fitting to the form given in Eq. (2) neglecting higher order terms. This introduces five fit parameters, namely $A_{00}, A_{01}, A_{11}$, $c_{1}$, and $\delta E_{1}$. We consider that excited states are sufficiently suppressed when the value extracted from the plateau method is consistent between two $t_{s}$, as well as with the other two approaches.

Computation of the three-point function.-The threepoint function $C_{3 \mathrm{pt}}\left(t_{s}, t_{\text {ins }}\right)$ receives contributions from two types of diagrams, one when the scalar operator couples to a valence quark inside the nucleon (connected) and one when it couples to a sea quark (disconnected). For $\sigma_{\pi N}$ both contributions are nonzero, while for $\sigma_{s}$ and $\sigma_{c}$ only the disconnected contribution is present. Evaluation of quark-disconnected contributions is notoriously difficult and it is only very recently that such contributions can be computed $[12-16,23,25]$. This is due to the fact that one needs to evaluate a closed quark loop of the form $\sum_{\vec{x}_{\text {ins }}} \operatorname{Tr}\left[G_{f}\left(x_{\text {ins }} ; x_{\text {ins }}\right)\right]$ where $G_{f}(x ; y)$ is the quark propagator. Because of the appearance of the sum over the spatial coordinate $\vec{x}_{\text {ins }}$, the evaluation of disconnected contributions requires knowledge of the quark propagator from all to all spatial coordinates, which translates into spatial volume $N_{S}^{3}$ inversions of the Dirac matrix compared to two per quark flavor required for the evaluation of the connected contribution. This is overcome by using 
stochastic techniques to evaluate the quark propagator entering the loop.

We use the twisted mass fermion discretization scheme [26], which, besides ensuring $O(a)$ improvement for physical observables after tuning to maximal twist [27], is particularly suited for evaluating scalar matrix elements. The first important advantage is that all scalar matrix elements are multiplicatively renormalizable [28] avoiding the mixing that occurs between the bare light and strange scalar quark matrix elements in other Wilson-type fermion discretizations. This property also holds for chiral invariant lattice formulations, which, however, are computationally much more demanding. The second important advantage has to do with the twisted mass term of the doublets in the action, which helps reduce the gauge noise of disconnected quark loops. This is because the isoscalar combination of a flavor doublet of the scalar operator transforms into an isovector of the pseudoscalar operator in the twisted mass formulation at maximal twist. For the $u$ - and $d$-flavor doublet we have $\bar{u} u+\bar{d} d=i \bar{\chi}_{u} \gamma_{5} \chi_{u}-i \bar{\chi}_{d} \gamma_{5} \chi_{d}$, where $\chi_{u}$ and $\chi_{d}$ are the two degenerate light quark fields in the twisted mass basis. The disconnected quark loop contribution to $\sigma_{\pi N}$ therefore becomes [29]

$$
\begin{aligned}
& \sum_{\vec{x}_{\text {ins }}} \operatorname{Tr}\left[i \gamma_{5} G_{\chi_{u}}\left(x_{\mathrm{ins}} ; x_{\mathrm{ins}}\right)-i \gamma_{5} G_{\chi_{d}}\left(x_{\mathrm{ins}} ; x_{\mathrm{ins}}\right)\right] \\
& \quad=2 \mu_{l} \sum_{y, \vec{x}_{\mathrm{ins}}} \operatorname{Tr}\left[\gamma_{5} G_{\chi_{u}}\left(x_{\mathrm{ins}} ; y\right) \gamma_{5} G_{\chi_{d}}\left(y ; x_{\mathrm{ins}}\right)\right] .
\end{aligned}
$$

The appearance of the small twisted light quark mass parameter $\mu_{l}$ allows for significant reduction in the gauge noise. In this form, stochastic techniques can be employed to obtain the trace via the so-called one-end trick [30] enabling the accurate computation of the quark loops at all time insertions $t_{\text {ins }}[13,31]$. Having the quark loop for all $t_{\text {ins }}$, the summation method can be employed without any further cost, which is an additional advantage of this formulation.

For the strange and the charm quarks a similar procedure can be followed. In this case we consider Osterwalder-Seiler doublets [32] to construct, in the twisted mass basis, the pseudoscalar current: $\frac{1}{2}\left(i \bar{\chi}_{f+} \gamma_{5} \chi_{f+}-i \bar{\chi}_{f-} \gamma_{5} \chi_{f-}\right)$, where $f=s, c$ and $f^{ \pm}$refers to taking $\pm \mu_{f}$. The nucleon matrix elements of these operators give $\sigma_{s}$ and $\sigma_{c}$. Unlike $\sigma_{\pi N}$, however, only purely disconnected contributions are involved.

Simulation parameters. - We use configurations simulated with the $N_{f}=2$ twisted mass fermion action [33] including a clover term with $c_{S W}=1.57551$ with $\mu_{l}$ tuned to obtained the physical value of the pion mass [34-38]. The lattice size is $N_{S}=48$ in the spatial and $N_{T}=96$ in the temporal direction. For the strange and charm doublets of Osterwalder-Seiler quarks, we tune the bare strange and charm quark twisted mass $a \mu_{s}$ and $a \mu_{c}$ to reproduce the experimental value of the $\Omega^{-}$and $\Lambda_{c}$ mass, respectively, obtaining $a \mu_{s}=0.0264(3)$ and $a \mu_{c}=0.3348(15)$.

The statistical uncertainty in the disconnected quantities is reduced as compared to that of Ref. [13] by increasing
TABLE I. $\quad N_{\text {cnf }}$ and $N_{\text {src }}$ is the number of configurations and source positions per configuration. For the disconnected loops, we give, in addition, the number of stochastic sources $N_{r}$, the number of high $\left(N_{r}^{\mathrm{HP}}\right)$ and low precision $\left(N_{r}^{\mathrm{LP}}\right)$ solves used within the TSM. For the two-point functions we used $N_{\mathrm{cnf}}=1800$ and $N_{\text {src }}=100$.

\begin{tabular}{lcccccccc}
\hline \hline \multicolumn{2}{l}{ Connected: three-point } & & \multicolumn{4}{c}{ Fermion loop } \\
\cline { 1 - 5 } \cline { 5 - 8 }$t_{s} / a$ & $N_{\mathrm{cnf}}$ & $N_{\text {src }}$ & & Quark flavor & $N_{\mathrm{cnf}}$ & $N_{r}^{\mathrm{HP}}$ & $N_{r}^{\mathrm{LP}}$ \\
\hline $10,12,14$ & 192 & 16 & & Light & 1800 & 2250 & 0 \\
16 & 265 & 88 & & Strange & 1800 & 63 & 1024 \\
18 & 517 & 88 & & Charm & 1800 & 5 & 1250 \\
\hline \hline
\end{tabular}

the statistics at reduced cost. This is accomplished by using a polynomially accelerated implicitly restarted Arnoldi method to obtain the $N_{e v}$ smallest eigenmodes of our linear system, which we use to precondition the conjugate gradient (CG) algorithm for successive solves on the same configuration. We take $N_{e v}=1400$, which yields a speedup of about twenty times as compared to the nondeflated CG. In the stochastic evaluation of the quark loops we use $Z(4)$ noise vectors with the number given in Table I. For the strange and charm quark loops, we use the truncated solver method (TSM), where we combine a large number of stochastic estimates computed to low precision with a small number of stochastic estimates computed to high precision, appropriately tuned $[39,40]$. Table I gives a summary of the statistics. All quark loop contributions are evaluated using graphics cards where the QUDA software is extended to include these quantities [41].

Results. - In Figs. 1 and 2 we show results for the ratio of Eq. (2) for the light, the strange, and the charm scalar operators, respectively. As $t_{s}$ increases the ratios converge in the plateau regions. We fit to the plateau when $t_{s}=1.7 \mathrm{fm}$ to extract results for $\sigma_{\pi N}$ and $\sigma_{s}$. For the charm sector, within the larger statistical errors, the plateaus are consistent already for $t_{s}=0.9 \mathrm{fm}$. We observe that the disconnected contribution to the $\sigma_{\pi N}$ is at the $10 \%$ level, a value consistent with the one found using an ensemble with pion mass of $373 \mathrm{MeV}$ [13].

The convergence of our results as we vary $t_{s}$ in the plateau method is demonstrated in Fig. 3 where we also show the results of the two-state fit and the summation method as we vary the lowest value of $t_{s}$ used in the fit, denoted by $t_{s}^{\text {low }}$. Our final value is taken from the plateau method after convergence with increasing $t_{s}$ is demonstrated, as well as when there is agreement with the two-state fit. The summation method has typically larger errors but it corroborates the plateau value. We quantify the systematic error due to excited state effects by weighting all values extracted from the plateau with the fit $p$ value for $t_{s} \geq 1.5 \mathrm{fm}(0.9 \mathrm{fm})$ for the light and strange (charm) $\sigma$ term and taking the variance.

Besides the systematic error due to the fit ranges, we have a systematic error from the determination of the lattice 


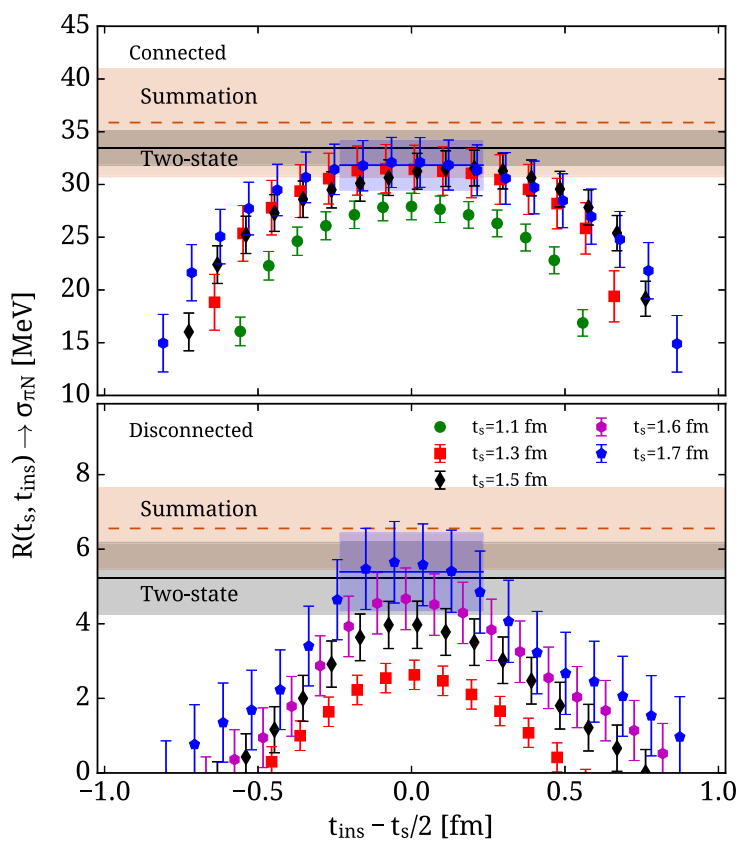

FIG. 1. Ratios yielding $\sigma_{\pi N}$, connected contribution (upper) and disconnected (lower), versus $t_{\text {ins }}$ shifted by half the sink-source time separation. The lines and associated bands show fits to the ratio in the plateau region (blue starting at the lower and finishing at the upper fit range used), the two-state fit (gray solid line) and the summation method (brown dashed line).

spacing, which we estimate by using the minimum and maximum values when different physical quantities are used to set the scale [34]. In addition, we estimate the finite volume correction on $\sigma_{\pi N}$ by evaluating the finite volume correction on the nucleon mass using baryon chiral perturbation theory [42], in combination with the Feynman-Hellmann theorem. We find a volume correction of $5 \%$ on $\sigma_{\pi N}$, and assume this to be an upper bound also for $\sigma_{s}$ and $\sigma_{c}$.

Conclusions. - Based on our analysis, the final values for $\sigma_{\pi N}, \sigma_{s}$, and $\sigma_{c}$ are

$$
\begin{aligned}
\sigma_{\pi N} & =37.2(2.6)_{\text {stat }}(1.9)_{\mathrm{exci}}(1.0)_{a}\left(\begin{array}{l}
1.8 \\
0.0
\end{array}\right)_{\mathrm{vol}} \mathrm{MeV}, \\
\sigma_{s} & =41.1(8.2)_{\mathrm{stat}}(4.7)_{\mathrm{exci}}(1.1)_{a}\left(\begin{array}{l}
2.0 \\
0.0
\end{array}\right)_{\mathrm{vol}} \mathrm{MeV} \\
\sigma_{c} & =79(21)_{\text {stat }}(6)_{\text {exci }}(2)_{a}\left(\begin{array}{l}
4 \\
0
\end{array}\right)_{\mathrm{vol}} \mathrm{MeV},
\end{aligned}
$$

and

where the first error is statistical and the rest are systematics due to excited states (exci), finite lattice spacing (a), and finite volume (vol) effects. The systematic errors are added linearly to arrive at the errors in the final values given in the abstract. Alternatively, we provide our results in terms of the dimensionless ratios, $f_{f}^{N}=\sigma_{f} / m_{N}$, obtaining

$f_{u d}^{N}=0.0399(28)\left(\begin{array}{l}40 \\ 21\end{array}\right), \quad f_{s}^{N}=0.0440(88)\left(\begin{array}{c}72 \\ 51\end{array}\right), \quad$ and

$f_{c}^{N}=0.085(22)\left(\begin{array}{c}11 \\ 7\end{array}\right)$,

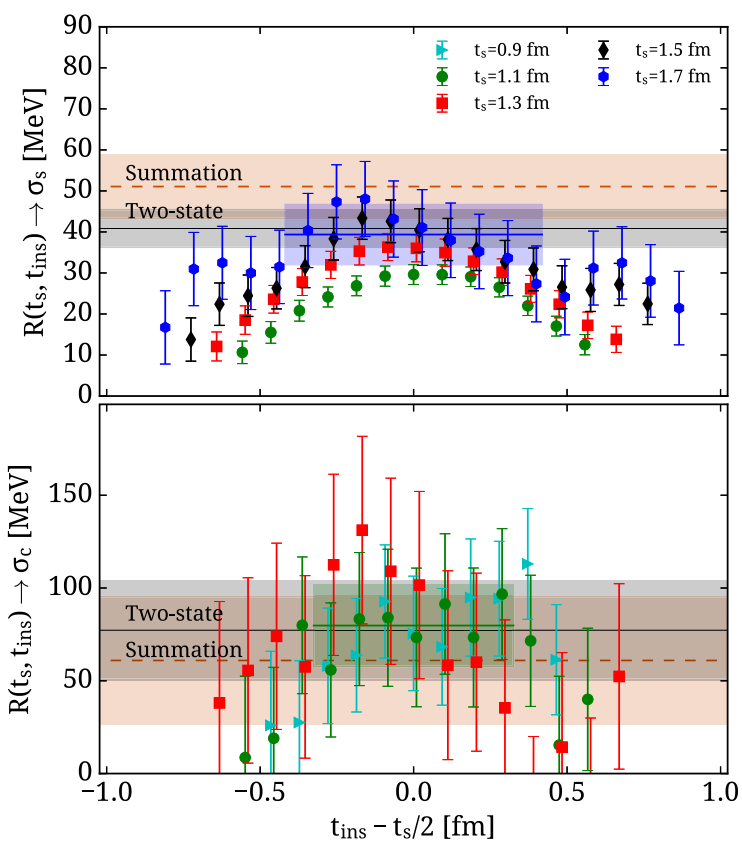

FIG. 2. The ratio from which $\sigma_{s}$ (upper) and $\sigma_{c}$ (lower) are extracted. The notation is the same as in Fig. 1.

using $m_{N}=933(8) \mathrm{MeV}$ [17], where the first error is statistical and the second is the sum of the systematic uncertainties due to the excited states and the finite volume. The isovector matrix element $\langle N|\bar{u} u-\bar{d} d| N\rangle$, has been

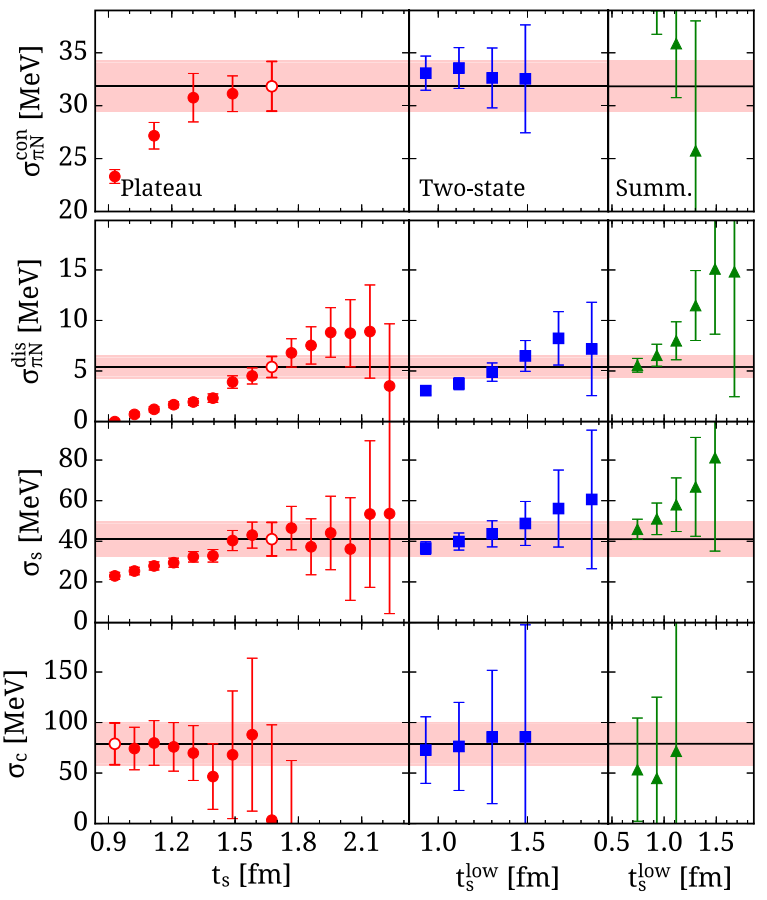

FIG. 3. Results for $\sigma_{f}$ from the plateau (left column), two-state (center column), and summation (right column) methods. From top to bottom we show the results for the connected and disconnected contributions to $\sigma_{\pi N}, \sigma_{s}$, and $\sigma_{c}$. $t_{s}^{\text {low }}$ is the smallest $t_{s}$ in the summation or two-state fits. The open symbol shows the selected final result and the red band its statistical error. 
computed [17] and combining it with the isoscalar matrix element we obtain the individual up- and down-quark contributions for the proton and the neutron in the isospin limit via

$f_{u}^{p}=\frac{2 m_{u d} r}{r+1} \frac{\langle N|\bar{u} u| N\rangle}{m_{N}}, \quad f_{u}^{n}=\frac{2 m_{u d} r}{r+1} \frac{\langle N|\bar{d} d| N\rangle}{m_{N}}$,

$f_{d}^{p}=\frac{2 m_{u d}}{r+1} \frac{\langle N|\bar{d} d| N\rangle}{m_{N}}, \quad f_{d}^{n}=\frac{2 m_{u d}}{r+1} \frac{\langle N|\bar{u} u| N\rangle}{m_{N}}$,

where $r=\left(m_{u} / m_{d}\right)=0.50(4)$ taken from Ref. [43]. We find

$f_{u}^{p}=0.0149(17)\left(\begin{array}{l}21 \\ 14\end{array}\right), \quad f_{u}^{n}=0.0117(15)\left(\begin{array}{l}18 \\ 12\end{array}\right)$,

$f_{d}^{p}=0.0234(23)\left(\begin{array}{l}27 \\ 16\end{array}\right), \quad$ and $f_{d}^{n}=0.0298(23)\left(\begin{array}{l}30 \\ 16\end{array}\right)$,

and for the $y_{N}$ parameter

$$
y_{N} \equiv \frac{2\langle N|\bar{s} s| N\rangle}{\langle N|\bar{u} u+\bar{d} d| N\rangle}=0.075(16)\left(\begin{array}{c}
14 \\
10
\end{array}\right) .
$$

Isospin breaking can be estimated by comparing the values of $f_{u / d}^{p / n}$ in Eq. (8) to those obtained when replacing the isovector matrix element with the neutron-proton mass splitting $\left.\quad \Delta m_{N}\right|_{\mathrm{QCD}}=2 m_{u d}(1-r / 1+r)\langle N|\bar{u} u-\bar{d} d| N\rangle$, and using the value $\left.\Delta m_{N}\right|_{\mathrm{QCD}}=2.52(17)(24) \mathrm{MeV}$ determined for nondegenerate $u$ - and $d$ - quarks [44]. We find a systematic error due to isospin breaking of maximum $1 \%$ on $f_{u / d}^{p / n}$, i.e., an order of magnitude smaller than the other errors.

In Fig. 4 we compare our values with recent results from phenomenology and lattice QCD omitting analyses that

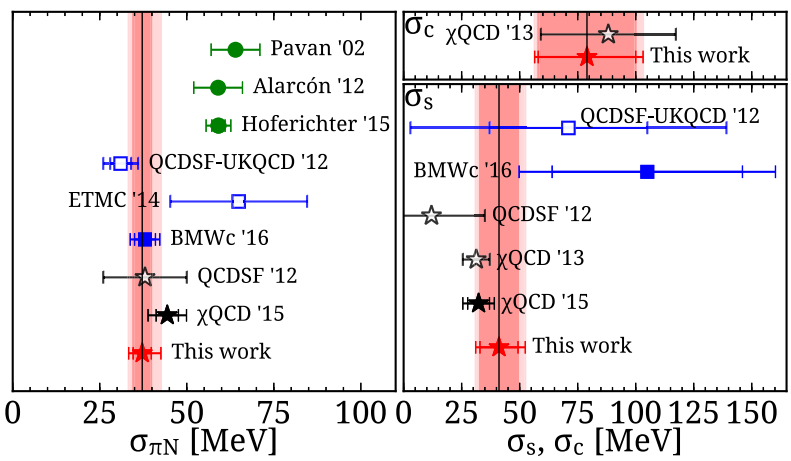

FIG. 4. Comparison of recent results for the $\sigma$ terms from phenomenology (filled circles) $[5,6,45]$ and from lattice QCD shown with squares using the Feynman-Hellmann theorem $[10,46,47]$ and asterisks using the direct method $[12,15,16]$. Open symbols use lattice QCD simulations that do not include the physical point. The smaller error bar is the statistical and the larger the total error. include simulations with pion masses larger than $500 \mathrm{MeV}$. As can be seen, our value for $\sigma_{\pi N}$ is in perfect agreement with the most recent value determined using the FeynmanHellmann theorem [10], which corroborates the consistency between the two methods. Such a value of $\sigma_{\pi N}$ is consistent with a small SU(3) breaking in conjunction with a small violation of the Okubo-Zweig-Iizuka (OZI) rule. However, there is tension with recent analyses based on the Roy-Steiner equations and experimental data on pionic atoms. The lattice $\mathrm{QCD}$ value implies a considerably smaller value of the $\pi N$-scattering lengths in disagreement with pionic-atom phenomenology. A thorough investigation of the lattice systematics, as well as an evaluation of the $\pi N$-scattering lengths within lattice QCD [48], can, thus, provide crucial input in resolving this puzzle.

We thank all members of ETMC for their cooperation. This work used computational resources from the Swiss National Supercomputing Centre (CSCS) under Projects No. s540 and No. s625, from the John von NeumannInstitute for Computing on the Juropa system and the BlueGene/Q system Juqueen at the research center in Jülich, from the Gauss Centre for Supercomputing on HazelHen (HLRS) and PRACE project access to the Tier- 0 computing resources Curie (CEA), Fermi (CINECA), and SuperMUC (LRZ). K. H. and Ch. K. acknowledge support from the Cyprus Research Promotion Foundation under Contract No. ТПЕ/П $\Lambda$ HРО/0311(BIE)/09.

[1] P. Cushman et al., in Proceedings of Community Summer Study 2013: Snowmass on the Mississippi (CSS2013) Minneapolis, $M N$ (2013).

[2] G. Bertone, D. Hooper, and J. Silk, Phys. Rep. 405, 279 (2005).

[3] J. R. Ellis, K. A. Olive, and C. Savage, Phys. Rev. D 77, 065026 (2008).

[4] J. Gasser, H. Leutwyler, and M. E. Sainio, Phys. Lett. B 253, 252 (1991).

[5] M. Hoferichter, C. Ditsche, B. Kubis, and U. G. Meissner, J. High Energy Phys. 06 (2012) 063.

[6] J. M. Alarcon, J. M. Camalich, and J. A. Oller, Phys. Rev. D 85, 051503 (2012).

[7] M. Hoferichter, J. Ruiz de Elvira, B. Kubis, and U.-G. Meiner, Phys. Rev. Lett. 115, 092301 (2015).

[8] H. Leutwyler, in Proceedings of the 8th International Workshop on Chiral Dynamics (CD 2015) Pisa, Italy (2015).

[9] R. D. Young and A. W. Thomas, Nucl. Phys. A844, 266c (2010).

[10] S. Durr et al., Phys. Rev. Lett. 116, 172001 (2016).

[11] W. Freeman and D. Toussaint (MILC Collaboration), Phys. Rev. D 88, 054503 (2013).

[12] M. Gong et al. (XQCD Collaboration), Phys. Rev. D 88, 014503 (2013).

[13] A. Abdel-Rehim, C. Alexandrou, M. Constantinou, V. Drach, K. Hadjiyiannakou, K. Jansen, G. Koutsou, and A. Vaquero, Phys. Rev. D 89, 034501 (2014). 
[14] C. Alexandrou, M. Constantinou, S. Dinter, V. Drach, K. Hadjiyiannakou, K. Jansen, G. Koutsou, and A. Vaquero, Phys. Rev. D 91, 094503 (2015).

[15] Y.-B. Yang, A. Alexandru, T. Draper, J. Liang, and K.-F. Liu, arXiv:1511.09089.

[16] G. S. Bali et al. (QCDSF Collaboration), Phys. Rev. D 85, 054502 (2012).

[17] A. Abdel-Rehim et al., Phys. Rev. D 92, 114513 (2015); 93, 039904(E) (2016).

[18] B. Blossier, P. Dimopoulos, R. Frezzotti, V. Lubicz, M. Petschlies, F. Sanfilippo, S. Simula, and C. Tarantino, Phys. Rev. D 82, 114513 (2010).

[19] N. Carrasco et al. (European Twisted Mass Collaboration), Nucl. Phys. B887, 19 (2014).

[20] B. Blossier et al. (ETM Collaboration), J. High Energy Phys. 07 (2009) 043.

[21] N. Carrasco et al., Phys. Rev. D 91, 054507 (2015).

[22] K. Takeda, S. Aoki, S. Hashimoto, T. Kaneko, J. Noaki, and T. Onogi (JLQCD Collaboration), Phys. Rev. D 83, 114506 (2011).

[23] H. Ohki, K. Takeda, S. Aoki, S. Hashimoto, T. Kaneko, H. Matsufuru, J. Noaki, and T. Onogi (JLQCD Collaboration), Phys. Rev. D 87, 034509 (2013).

[24] G. Martinelli and C. T. Sachrajda, Phys. Lett. B 196, 184 (1987).

[25] R. Babich, R. C. Brower, M. A. Clark, G. T. Fleming, J. C. Osborn, C. Rebbi, and D. Schaich, Phys. Rev. D 85, 054510 (2012).

[26] R. Frezzotti, P. A. Grassi, S. Sint, and P. Weisz (Alpha Collaboration), J. High Energy Phys. 08 (2001) 058.

[27] R. Frezzotti and G. C. Rossi, J. High Energy Phys. 08 (2004) 007.

[28] S. Dinter, V. Drach, R. Frezzotti, G. Herdoiza, K. Jansen, and G. Rossi (ETM Collaboration), J. High Energy Phys. 08 (2012) 037.

[29] C. Michael and C. Urbach (ETM Collaboration), Proc. Sci., LAT2007 (2007) 122.

[30] C. McNeile and C. Michael, Phys. Rev. D 73, 074506 (2006).
[31] C. Alexandrou, M. Constantinou, V. Drach, K. Hadjiyiannakou, K. Jansen, G. Koutsou, A. Strelchenko, and A. Vaquero, Comput. Phys. Commun. 185, 1370 (2014).

[32] R. Frezzotti and G. C. Rossi, J. High Energy Phys. 10 (2004) 070.

[33] K. Jansen and C. Urbach, Comput. Phys. Commun. 180, 2717 (2009).

[34] A. Abdel-Rehim et al. (ETM Collaboration), arXiv: 1507.05068 .

[35] A. Abdel-Rehim et al., Proc. Sci., LATTICE2014 (2015) 119 [arXiv:1411.6842].

[36] A. Abdel-Rehim, F. Burger, A. Deuzeman, K. Jansen, B. Kostrzewa, L. Scorzato, and C. Urbach, Proc. Sci., LATTICE2013 (2014) 414 [arXiv:1311.5495].

[37] A. Abdel-Rehim et al., Proc. Sci., LATTICE2013 (2014) 264 [arXiv:1311.4522].

[38] A. Deuzeman, K. Jansen, B. Kostrzewa, and C. Urbach, Proc. Sci., LATTICE2013 (2014) 416 [arXiv:1311.4521].

[39] G. S. Bali, S. Collins, and A. Schafer, Comput. Phys. Commun. 181, 1570 (2010).

[40] C. Alexandrou, K. Hadjiyiannakou, G. Koutsou, A. O'Cais, and A. Strelchenko, Comput. Phys. Commun. 183, 1215 (2012).

[41] C. Alexandrou, K. Hadjiyiannakou, G. Koutsou, A. Strelchenko, and A. V. Avils-Casco, Proc. Sci., LATTICE2013 (2014) 411 [arXiv:1401.6750].

[42] A. Ali Khan et al. (QCDSF-UKQCD Collaboration), Nucl. Phys. B689, 175 (2004).

[43] S. Aoki et al., Eur. Phys. J. C 74, 2890 (2014).

[44] S. Borsanyi et al., Science 347, 1452 (2015).

[45] M. M. Pavan, I. I. Strakovsky, R. L. Workman, and R. A. Arndt, PiN Newslett. 16, 110 (2002).

[46] R. Horsley, Y. Nakamura, H. Perlt, D. Pleiter, P. E. L. Rakow, G. Schierholz, A. Schiller, H. Stüben, F. Winter, and J.M. Zanotti (QCDSF-UKQCD Collaboration), Phys. Rev. D 85, 034506 (2012).

[47] C. Alexandrou, V. Drach, K. Jansen, C. Kallidonis, and G. Koutsou, Phys. Rev. D 90, 074501 (2014).

[48] J. M. Alarcón (private communication). 